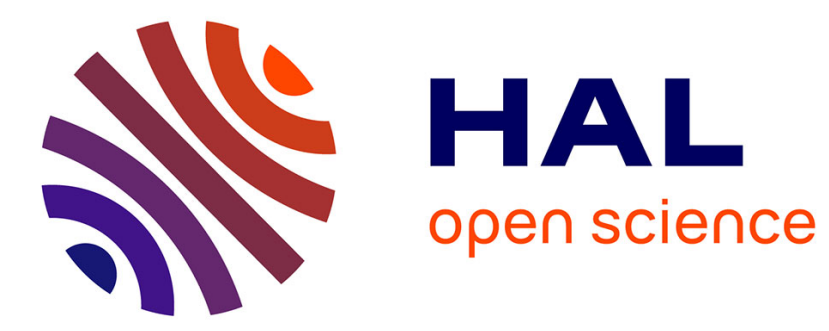

\title{
Influence des défauts de surface sur le comportement des MESFET GaAs
}

\author{
C. Le Mouellic, S. Mottet, J.-M. Dumas, D. Lecrosnier
}

\section{To cite this version:}

C. Le Mouellic, S. Mottet, J.-M. Dumas, D. Lecrosnier. Influence des défauts de surface sur le comportement des MESFET GaAs. Revue de Physique Appliquée, 1984, 19 (2), pp.149-154. 10.1051/rphysap:01984001902014900 . jpa-00245168

\section{HAL Id: jpa-00245168 https://hal.science/jpa-00245168}

Submitted on 1 Jan 1984

HAL is a multi-disciplinary open access archive for the deposit and dissemination of scientific research documents, whether they are published or not. The documents may come from teaching and research institutions in France or abroad, or from public or private research centers.
L'archive ouverte pluridisciplinaire HAL, est destinée au dépôt et à la diffusion de documents scientifiques de niveau recherche, publiés ou non, émanant des établissements d'enseignement et de recherche français ou étrangers, des laboratoires publics ou privés. 
Classification

Physics Abstracts

73.00

\title{
Influence des défauts de surface sur le comportement des MESFET GaAs
}

\author{
C. Le Mouellic, S. Mottet, J.-M. Dumas et D. Lecrosnier \\ CNET Lannion B, Division ICM, Département TOH, BP 40, 22301 Lannion Cedex, France
}

(Reçu le 29 juillet 1983, révisé le 14 octobre, accepté le 21 octobre 1983)

\begin{abstract}
Résumé. - L'étude de la fiabilité de MESFET GaAs passivés avec une couche de silice nous a permis de mettre en évidence un phénomène parasite associé aux dégradations des performances hyperfréquences. Ce phénomène apparaît sur le réseau de caractéristiques $I_{\mathrm{DS}}\left(V_{\mathrm{DS}}\right)$ sous la forme d'un coude du courant en régime de saturation. Ce phénomène s'accentue avec le vieillissement du transistor et nous montrons qu'il peut être lié au comportement de la surface de $\mathrm{GaAs}$. Une simulation numérique nous a permis d'interpréter ce phénomène par la présence d'états de surface dans les zones d'accès de ces transistors.
\end{abstract}

\begin{abstract}
Reliability studies of $\mathrm{SiO}_{2}$ passivated GaAs MESFET have revealed an anomalous phenomena associated with the degradation of high frequencies performances. This phenomena appears on the current-voltage characteristics which exhibit a " kink " when devices are operating in the saturation region. This kink increases when the devices are submitted to life tests, and we have found it is related to the surface of GaAs. A model is proposed to explain these anormal $I_{\mathrm{DS}}\left(V_{\mathrm{DS}}\right)$ characteristics : it is based on the existence of surface states present between gate and drain and gate and source.
\end{abstract}

\section{Introduction.}

Sur des transistors MESFET GaAs passivés avec une couche de silice, nous avons observé une anomalie dans les caractéristiques courant tension $I_{\mathrm{DS}}\left(V_{\mathrm{DS}}\right)$. Cette anomalie se traduit par un coude du courant en

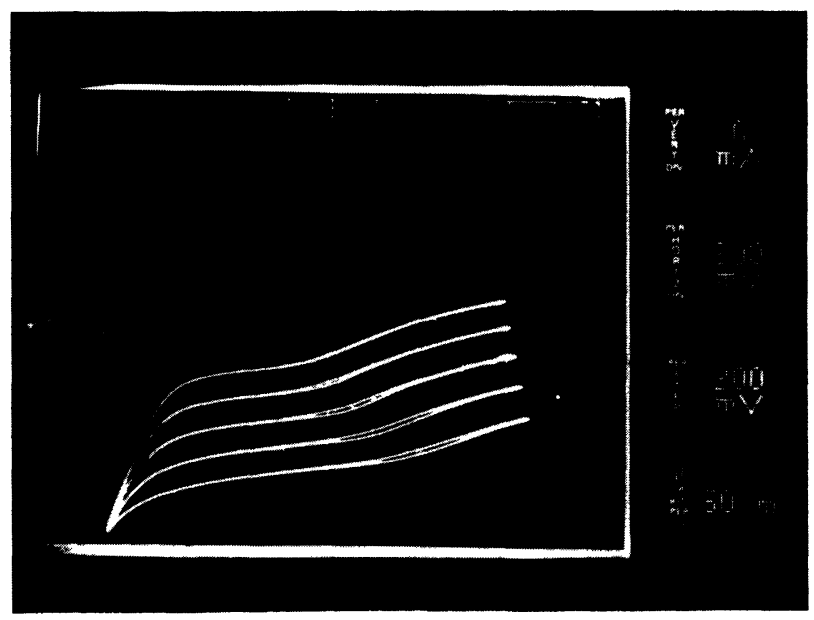

Fig. 1. - Caractéristique courant/tension de drain avec le phénomène de coude.

[Drain current/voltage characteristics with the "kink » phenomena.] régime de saturation ainsi que le montre la figure 1. Tihanyi et Schlotterer [1] ont observé des caractéristiques voisines sur des transistors MOS sur silicium, et l'ont attribué à des effets d'avalanche dans la couche active. De même, sur des MESFETs GaAs, Goronkin et Vaitkus [2] ont tenté d'expliquer cette anomalie en l'associant à une ionisation par impact à l'interface couche active substrat semi-isolant. T. Nozaki et al. [3] observent que cette anomalie est considérablement réduite par la présence d'une couche tampon de type $\mathbf{n}$ très peu dopée $\left(10^{13} \mathrm{~cm}^{-3}\right)$. Enfin, T. Nozaki et al. [4] et W. T. Anderson [5] constatent qu'une couche tampon dopée de type $\mathrm{p}$ réduit l'hystérésis des caractéristiques $I_{\mathrm{DS}}\left(V_{\mathrm{DS}}\right)$. Nous voyons donc que ces effets ont été jusqu'à présent attribués à l'interface couche active-substrat. Les résultats, que nous présentons ici, mettent plutôt en cause la surface de ces composants.

\section{Résultats expérimentaux.}

2.1 VIEILliSSEMENT ACCÉLÉRÉ. RÔLE DE LA SURFACE. Cet essai a été effectué sur des MESFETs faible bruit dont la structure est représentée sur la figure 2. Ces transistors sont passivés à l'aide d'une couche de $2000 \AA \mathrm{de}^{\mathrm{SiO}}{ }_{2}$ déposée par CVD basse température $\left(\simeq 380^{\circ} \mathrm{C}\right)$. Pendant le vieillissement, les transistors 


\section{STRUCTURE DU MESFET}

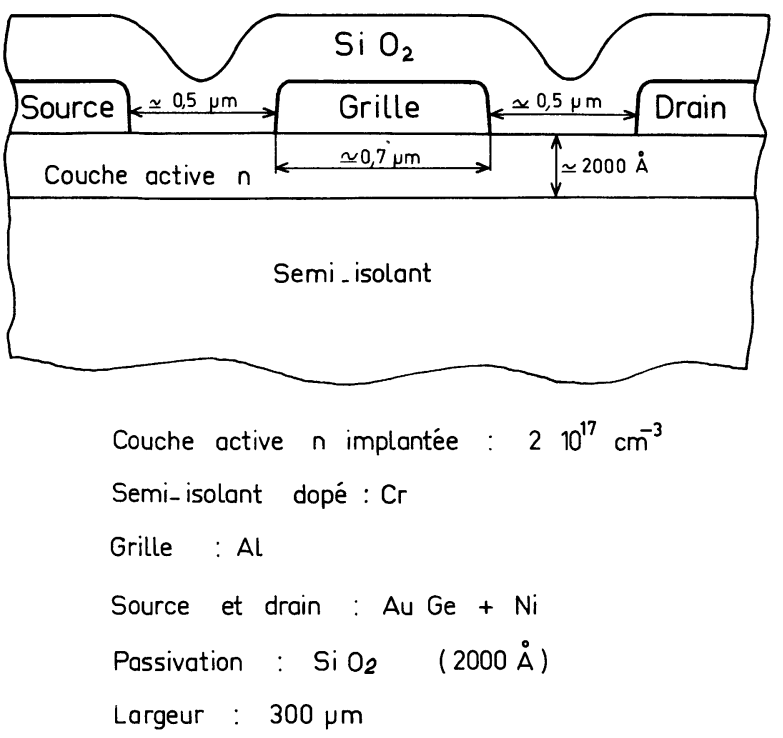

Fig. 2. - Structure de MESFET GaAs passivé avec $\mathrm{SiO}_{2}$. [Structure of MESFET passivated with $\mathrm{SiO}_{2}$.] fonctionnaient dans les conditions suivantes : $V_{\mathrm{DS}}=3 \mathrm{~V}, I_{\mathrm{DS}}=10 \mathrm{~mA}$, température de canal $=160^{\circ} \mathrm{C}$. L'ensemble des résultats apparaît sur la figure 3. On constate qu'après $500 \mathrm{~h}$, les performances hyperfréquences (gain, facteur de bruit mesurés à la fréquence de $11 \mathrm{GHz}$ ) se sont notablement dégradées. On observe aussi une augmentation du décrochement sur le réseau $I_{\mathrm{Ds}}\left(V_{\mathrm{DS}}\right)$ qui correspond à une baisse sensible du courant de saturation pour des tensions $V_{\mathrm{D}}$ inférieures à 2 V. Après avoir enlevé la silice, par étapes successives, avec une solution chimique $\mathrm{FNH}_{4}: \mathrm{HF}$ : $\mathrm{H}_{2} \mathrm{O}(6: 1: 7)$ qui n'attaque pas les métallisations, nous avons retrouvé pratiquement les performances hyperfréquences initiales et un réseau de caractéristiques quasi normal. Notons que cette "guérison » n'apparaît que lorsque la dernière couche de silice est enlevée. De plus, il est possible que pendant cette attaque, nous ayons enlevé une couche superficielle de GaAs - cette couche pouvant éventuellement être constituée d'oxyde naturel de GaAs.

Cette expérience de gravure de la couche de passivations montre clairement que la surface de GaAs située entre grille-drain et/ou grille-source joue un rôle important sur l'anomalie que nous avons observée.
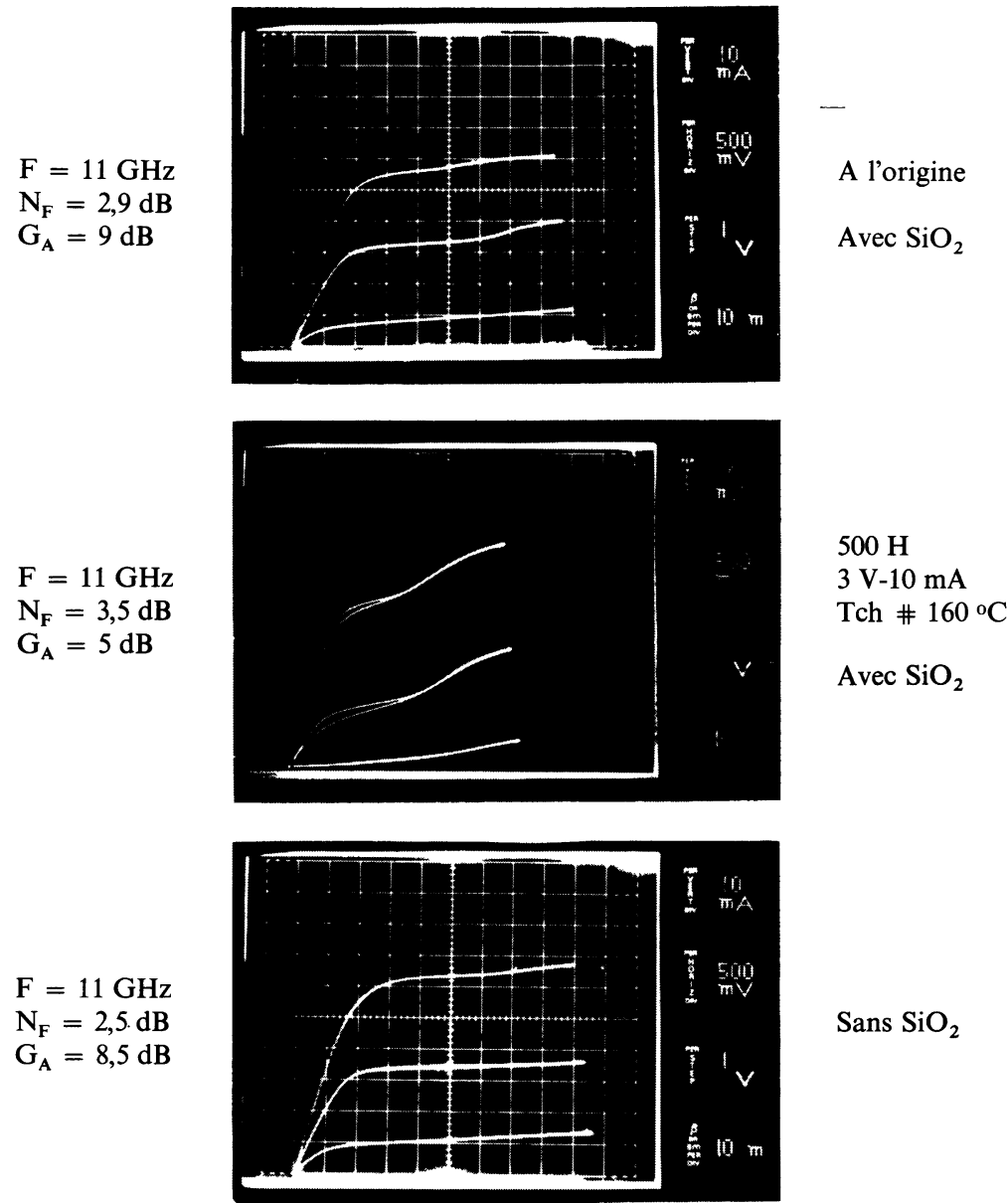

Sans $\mathrm{SiO}_{2}$

Fig. 3. - Caractéristique courant/tension de drain, $a$ ) à l'origine, $b$ ) après vieillissement, $c$ ) après décapage du $\mathrm{SiO}_{2}$.

[Drain current/voltage characteristics, $a$ ) at $t=0, b)$ after aging, $c$ ) after etching of $\mathrm{SiO}_{2}$.] 


\section{2 CARACTÉRISATION ÉLECTRIQUE.}

2.2.1 Influence de la fréquence de balayage. - Sur la figure $4 a$ nous avons représenté des courbes $I_{\mathrm{DS}}\left(V_{\mathrm{DS}}\right)$ pour $V_{\mathrm{g}}=0 \mathrm{~V}$ et $V_{\mathrm{g}}=-0,5 \mathrm{~V}$. Ces mesures ont été obtenues avec une vitesse de balayage de $V_{\mathrm{Ds}}$ lente. On constate alors un décrochement à l'aller et une caractéristique normale au retour, ce qui fait apparaître
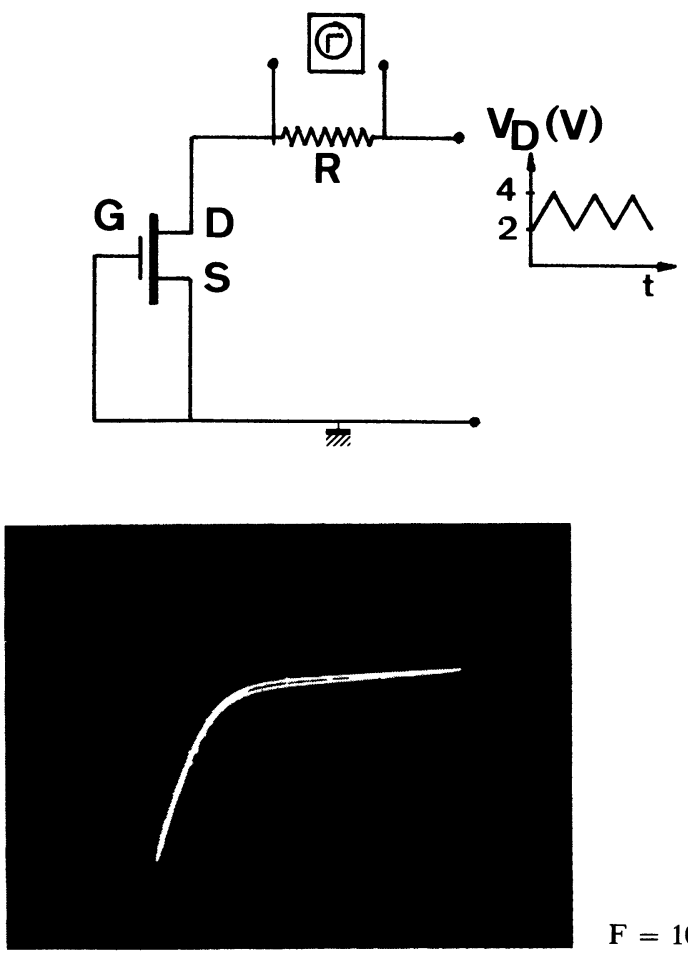

$F=10^{4} \mathrm{~Hz}$

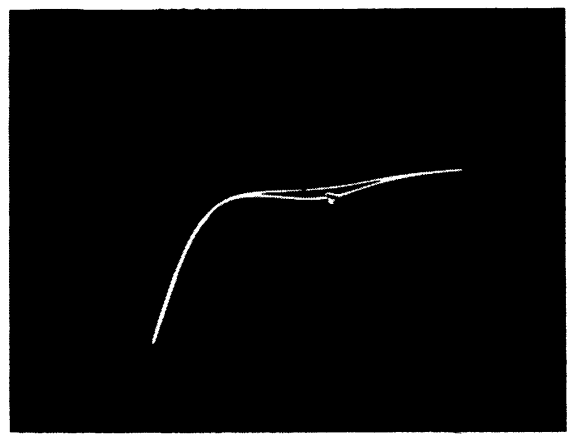

$\mathbf{F}=0,1 \mathrm{~Hz}$

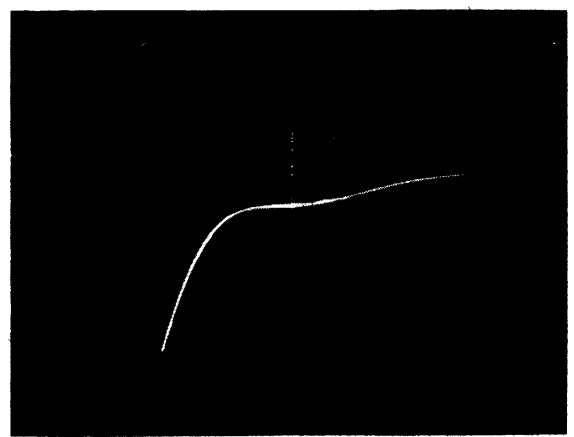

$F=0,01 \mathrm{~Hz}$

Fig. 4. - Caractéristiques courant/tension de drain en fonction de la fréquence de balayage.

[Drain current voltage characteristics versus scan frequency.] un effet d'hystérésis. A l'aide du montage représenté sur la figure 4 nous avons étudié sur un transistor l'influence de la fréquence de balayage de $V_{\text {Ds. }}$ Pour les fréquences élevées $\left(10^{4} \mathrm{~Hz}\right)$ on peut remarquer un comportement quasi normal du courant de saturation. Pour $F=0,1 \mathrm{~Hz}$ on observe le phénomène de décrochement et d'hystérésis. Par contre pour un balayage très lent $(0,01 \mathrm{~Hz})$, le décrochement s'accentue alors que l'hystérésis disparaît. Ces résultats font donc apparaître une dépendance fréquencielle du phénomène. Notons qu'elle a déjà été observée par $S$. Makram-Eheid et al. [6] dans l'étude de la commutation en deux temps et par M. Ozeki et al. [7] dans le comportement de la transconductance.

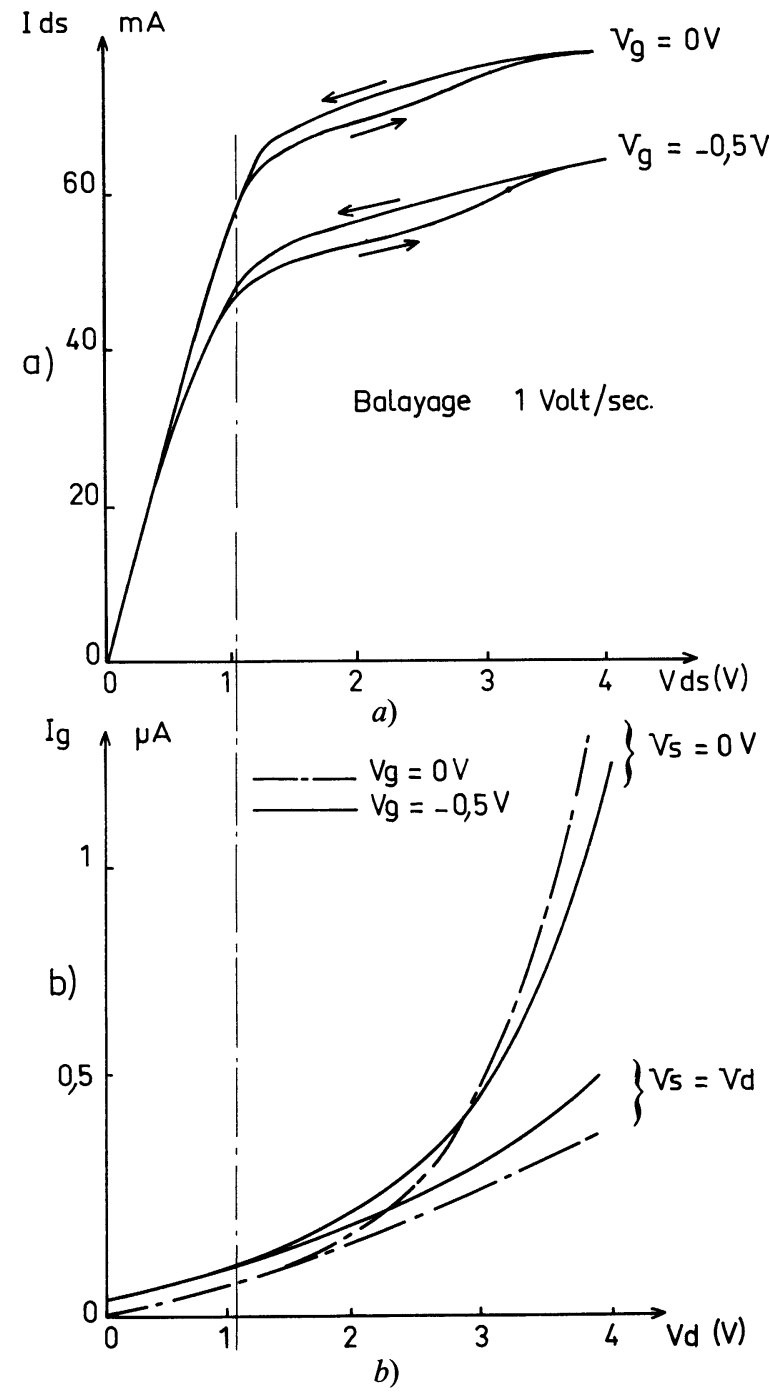

Fig. 5. - a) Caractéristique courant/tension de drain, b) Courant de grille en fonction de la tension de drain.

[a) Drain current/voltage characteristics, b) gate current versus drain voltage.]

2.2.2 Courant de grille. - Sur la figure $5 b$ nous avons porté le courant de fuite de grille en fonction de $V_{\mathrm{DS}}$ pour deux valeurs de $V_{\mathrm{g}}\left(V_{\mathrm{g}}=0 \mathrm{~V}\right.$ et $\left.-0,5 \mathrm{~V}\right)$. Lorsque source et drain sont connectés ensemble, 
on constate une croissance régulière du courant en fonction de la tension appliquée. Lorsque le transistor est polarisé normalement (source à la masse; $V_{\mathrm{GS}}=0 \mathrm{~V} ; V_{\mathrm{DS}}$ positif) les caractéristiques sont très différentes. Pour $V_{\mathrm{D}}$ inférieure à la tension de saturation, le courant est identique aux valeurs précédentes, par contre dans la zone de saturation du transistor le courant augmente notablement et pour $V_{\mathrm{D}} \simeq 3 \mathrm{~V}$ les caractéristiques mesurées pour $V_{\mathrm{g}}=0 \mathrm{~V}$ et $V_{\mathrm{g}}=-0,5 \mathrm{~V}$ se croisent. Un tel comportement qui a été observé par R. Yamamoto [7] est jusqu’à présent mal expliqué.

\section{Modélisation.}

Il est couramment admis que pour GaAs, les états de surface bloquent le niveau de Fermi en milieu de bande interdite ce qui a pour effet de créer une zone de charge d'espace dans les zones d'accès du transistor MESFET [8, 9]. La modélisation numérique que nous avons développée prend en compte des zones de charge d'espace situées entre grille-drain et grille-source. Les équations à résoudre sont :

$\operatorname{div} \varepsilon(\operatorname{grad} \varphi)=q(n-d o p) . \quad$ Equation de Poisson

$\frac{1}{q} \operatorname{div}(\mathbf{J n})=0 . \quad$ Equation de conservation du courant

$\operatorname{avec} \mathbf{J n}=\mu_{n}(n q \operatorname{grad} \varphi-k T \operatorname{grad} n)$.
Dans ces expressions, les densités de trous et les termes de génération-recombinaison ont été négligés :

- dop représente la densité locale d'impuretés ionisées.

- $\mu_{n}$ la mobilité dépendante du champ électrique prenant en compte les phénomènes de vitesse limite.

$-\varphi$ le potentiel et $n$ la densité de porteurs.

En ce qui concerne la densité des charges de surfaces nous avons considéré une très faible couche de matériau $(20 \AA)$ dans laquelle sont localisées des charges acceptrices ionisées. Cette densité est le seul paramètre variable de la modélisation.

La simulation porte sur le transistor de la figure 2 . Nous avons pris pour la hauteur de barrière Schottky une valeur de $0,8 \mathrm{eV}$. Les résultats présentés sont donnés pour une tension drain de $2 \mathrm{~V}$ (régime saturation).

Sur la figure $6 a$ et $b$ sont représentées respectivement les équipotentielles et la densité de porteurs dans la couche active lorsque la densité de charges de surface est nulle. On remarque la présence de la zone déplétée sous la grille et l'effet d'accumulation et de manque d'électrons dans la zóne de pincement. Dans ce cas, le courant calculé $I_{\text {Ds }}$ est de $70 \mathrm{~mA}$.

La figure 7 montre l'effet de la présence d'une densité $N \mathrm{~s}=10^{12} \mathrm{~cm}^{-2}$ charges en surface. On voit apparaître une zone déplétée dans les zones d'accès du transistor et le courant calculé $I_{\mathrm{DS}}$ est de $67 \mathrm{~mA}$.
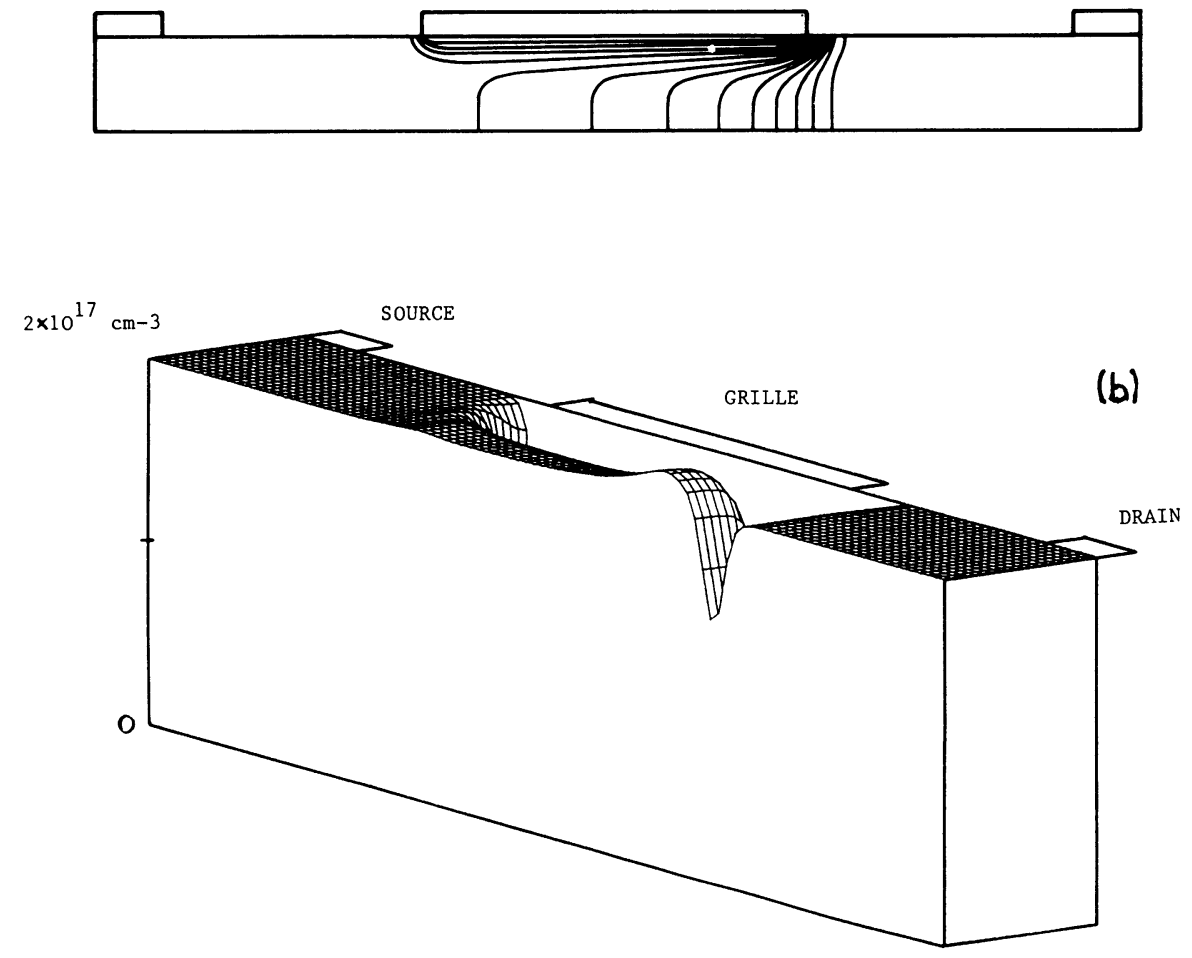

Fig. 6. - Simulation sans états de surface, $a$ ) équipotentielles, $b$ ) densité de porteurs.

[Simulation without surface state $: a$ ) equipotential lines, $b$ ) carriers density.] 

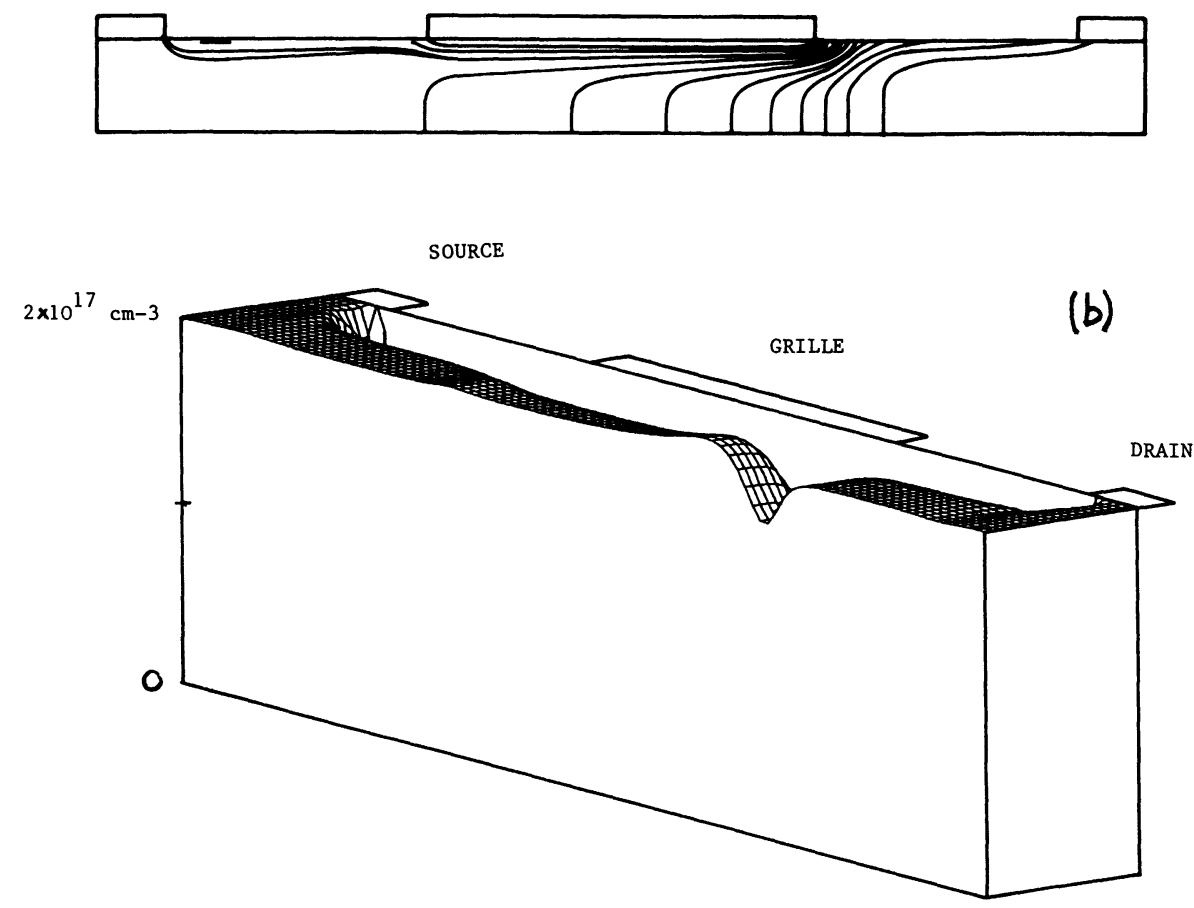

Fig. 7. - Simulation avec états de surface, $a$ ) équipotentielles, $b$ ) densité de porteurs.

[Simulation with surface states, $a$ ) equipotential line, $b$ ) carriers density.]

Une simulation faite avec une densité de charges de $2 \times 10^{12} \mathrm{~cm}^{-2}$ a entraîné une baisse du courant de saturation de $15 \mathrm{~mA}$. Ainsi, il apparaît qu'une densité relativement élevée de charges de surface peut conduire à expliquer la variation expérimentale du courant de saturation.

\section{Discussion.}

D'après la figure $4 b$, on constate que le régime de saturation du transistor provoque un excès du courant de fuite de grille. Cet excès peut venir d'un courant de trous comme le suggère R. Yamamoto [7]. L'origine de ces trous est encore mal expliquée et nous semble liée aux anomalies de distribution de porteurs et de champ électrique dans la zone de pincement du canal : si on considère cette région en régime de saturation, les électrons sont en vitesse limite; les trous ne le sont pas. La conservation du courant de trous mène à une forte diminution de la densité de ces porteurs et dans cette région on a $n p<n_{i}^{2}$. Ceci implique une génération possible de paires électron-trou. Enfin, un effet de multiplication semble également intervenir lorsque la tension drain est élevée $\left(V_{\mathrm{Ds}}>3 \mathrm{~V}\right)$.

Ces trous, ainsi créés, donnent naissance au courant de grille, mais peuvent également être collectés par le champ transverse existant dans les zones d'accès $\mathrm{du}$ transistor et se piègent en surface. Ceci provoque alors une réduction de la charge d'espace dans ces régions et donc une augmentation du courant de drain comme le montre la simulation.
La surface de GaAs se comporte donc comme un puits de potentiel pour les trous. Le coude des caractéristiques $I_{\mathrm{DS}}\left(V_{\mathrm{DS}}\right)$ apparaît lorsque la densité des trous générés dans le canal est suffisante pour permettre le remplissage de ce puits.

Quant à l'hystérésis, elle est attribuée à la cinétique de réémission des trous piégés en surface. Le remplissage est supposé instantané. Les courbes de la figure 4 peuvent être interprétées de la manière suivante :

- aux fréquences de balayage élevées $\left(F=10^{4} \mathrm{~Hz}\right)$, les pièges ne peuvent se vider, il n'y a pas de variation de la charge d'espace des zones d'accès du transistor : la caractéristique $I_{\mathrm{DS}}\left(V_{\mathrm{DS}}\right)$ ne présente pas d'anomalie ; - aux fréquences très basses $(F=0,01 \mathrm{~Hz})$, le dépiégeage est possible, la variation des zones de charge d'espace provoque le coude ;

- aux fréquences intermédiaires $(F \simeq 1 \mathrm{~Hz})$, la réémission des pièges est incomplète et conduit à une hystérésis.

\section{Conclusion.}

Les résultats présentés dans cette étude mettent en évidence le rôle de la surface de GaAs sur les anomalies (coude et hystérésis) des caractéristiques $I_{\mathrm{DS}}\left(V_{\mathrm{DS}}\right)$ de transistor MESFET passivés avec de la silice. Ces anomalies sont associées à la présence de pièges à la surface des zones d'accès des transistors.

Des essais de vieillissement montrent une évolution avec le temps des surfaces ainsi passivées conduisant à une dégradation des performances hyperfréquences. 


\section{Bibliographie}

[1] Tihanyi, J., SChlotterer, H., Influence of the floating substrate potential on the characteristics of ESFI MOS transistors, Solid State Electron. 18 (1975) 309-314.

[2] Goronkin, H., Vaitkus, R. L., Impact ionization of traps in ion implanted GaAs MESFET's Inst. Phys. Conf. Ser. No 63, chapter 6, Int. Symp., GaAs Related Compounds, Japan 1981, p. 287-292.

[3] Nozaki, T., Ogama, M., Terao, H., Watabane, W., Multilayer epitaxial technology for the Schottkybarrier GaAs field-effect transistor, $\backslash$ Inst. Phys. Conf. Ser. No 24, chapter 2, Gallium Arsenide and Related Compounds, 1974, p. 46-54.

[4] Nozaki, T., Ohata, K., Sub-micron gate GaAs MESFET's with Ion-implanted channels, Proceedings of the 8th Conference on Solid State Devices (1976).

[5] Anderson, Jr, W. T., Simons, M., King, E. E. DieTRICH, H. B., LAMBERT, R. J., Reduction of long term transient radiation response in ion implanted $\mathrm{GaAs}$ Fets, IEEE Trans. Nucl. Sci., NS-29, № 6 (1982).
[6] Makram-Ebeid, S., Mitonneau, A., Laurence, G., Low Frequency Anomalies in GaAs MESFET Structures, Semi-insulating III-V, Evian 1982, p. 336-343.

[7] Ozeki, M., Kodoma, K., Takikawa, M., Shibatomi, A., Analysis of electrical and optical properties of insulating film GaAs interfaces using MESFET type structures, J. Vac. Sci. Techn. 21 (2) 1982.

[8] Yamamoto, R., Higashisaka, A., Hasegana, F., Light Emission and Burnout characteristics of GaAs Power MESFET's, IEEE Trans. Electron. Devices ED-25 (1978) 567-573.

[9] HaRIU, T., Theoretical calculation of Normally-off GaAs MESFET characteristics including effects of surface depletion layer, Japan. J. Appl. Phys. 21 № 2 (1982) L77-L79.

[10] Spicer, W. E., Lindau, I., Skeath, P., Su, C. Y., J. Vac. Sci. Technol. 17 (1980) 1019. 